\title{
Indonesian license plate recognition with improved horizontal-vertical edge projection
}

\author{
Ida Nurhaida, Imam Nududdin, Desi Ramayanti
}

Faculty of Computer Science, Universitas Mercu Buana, Indonesia

\begin{tabular}{l}
\hline \hline Article Info \\
\hline Article history: \\
Received Feb 26, 2020 \\
Revised Apr 7, 2020 \\
Accepted May 6, 2020 \\
\hline
\end{tabular}

\section{Keywords:}

Computer vision

Image processing

License plate recognition

Morphology operation

\begin{abstract}
License plate recognition (LPR) is one of the classical problems in the field of object recognition. Its application is very crucial in the automation of transportation system since it helps to recognise a vehicle identity, which information is stored in the license plate. LPR usually consists of three major phases: pre-processing, license plate localisation, optical character recognition (OCR). Despite being classical, its implementation faced with much more complicated problems in the real scenario. This paper proposed an improved LPR algorithm based on modified horizontal-vertical edge projection. The method uses for detecting and localising the region of interest. It is done using the horizontal and vertical projection of the image. Related works proved that the modified horizontal-vertical edge projection is the simplest method to be implemented, yet very effective against Indonesian license plate. However, its performance gets reduced when specular reflection occurs in the sample image. Therefore, morphological operations are utilised in the pre-processing phase to reduce such effects while preserving the needed information. Eighty sample images which captured using various camera configurations were used in this research. Based on the experimental results, our proposed algorithm shows an improvement compared with the previous study and successfully detect 71 license plates in 80 image samples which results in $88.75 \%$ accuracy.
\end{abstract}

This is an open access article under the CC BY-SA license.

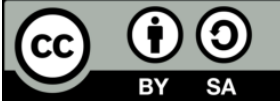

\section{Corresponding Author:}

Ida Nurhaida

Faculty of Computer Science

Universitas Mercu Buana

Jl. Meruya Selatan no. 1, Indonesia

Email: ida.nurhaida@mercubuana.ac.id

\section{INTRODUCTION}

Object recognition is one of the most interesting fields among the computer vision researcher since it's applicable in many sectors of our everyday life. One of the classical problem that still relevant even today was license plate recognition, because of the many challenges that must be tackled such as variations of the license plate characteristics in each country around the world, noise in the image, environmental factors, etc. Many researchers around the world have developed many algorithms for license plate recognition. Still, due to the limited sample, most of them are crafted only for a specific country with specific license plate characteristics (background colour, format, font type, etc.). They might not work for other countries with different license plate characteristics.

License plate in Indonesia has its unique characteristics. It has a black coloured background with a white border and text. The usage of unique alphabet code to differentiate license plates across the regions in Indonesia, also the license plate is structured based on a standardised format. The details are described within 
the Figure 1. License plate recognition usually consists of three major phases: pre-processing, license plate localisation, and optical character recognition (OCR). Pre-processing is a phase where the input image will be prepared, enhanced, and optimised for further processing [1, 2]. According to [3-5], license plate localisation is a phase in which the vehicle license plate area is to be determined, localised, and segmented out of the original picture to isolate the license plate from the other objects which considered as noise. After the license plate has been segmented and isolated, the next phase will be character recognition, where all of the characters within the license plate will be extracted and processed by some sort of algorithm, usually template matching.

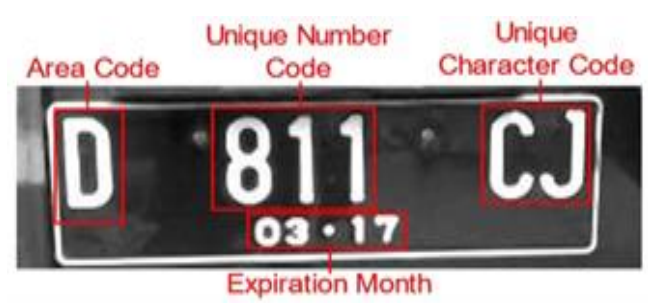

Figure 1. Indonesian license plate standardised format

The study [3, 4] modified the algorithm to adapt to Indonesian license plate characteristics, and it approves to be effective. Those papers have reviewed and summarised techniques for license plate recognition in Indonesia throughout recent years. The papers consist of a short description of the reviewed algorithms and also the comparison between each algorithm. Despite being effective, the algorithm still struggles when it faced with specular reflection or object with high saturation intensities. Hidayatullah, et al., [3] introduce an algorithm that is heavily influenced by [5] but with a better approach to adapt to the Indonesian license plate. It shows that the algorithm is pretty useful in detecting Indonesian license plate with 63 detected license plate out of 80 sample images. The researcher also stated that one of the key problems which cause the detection to be a failure is specular reflection. Devapriya et al., [5] proposed a license plate recognition algorithm for the Indian license plate that rely heavily on the morphological operation. Another approach of license plate recognition is presented by Gohil [6]. Research [6] proposed a simple yet effective algorithm to be used for Californian license plate recognition. The algorithm works by detecting the area of vehicle license plate based on the horizontal edge and vertical edge projection. It is the most straightforward yet very useful algorithm for license plate recognition. However, this is not very simple to implement this algorithm for the Indonesian license plate. It is hard to expose such rectangular object because Indonesian license plate has a black background, and sometimes the white border is not visible due to the lighting condition or get obstructed by another object. Therefore, it will be very prone to misdetection. We believe something could be done to improve the performance of the algorithm.

\section{RELATED WORKS}

Various research for license plate recognition has been introduced using many algorithms. According to the paper, it seems like many researchers have been utilising morphology operation and edge detection technique to construct their license plate recognition algorithm. Moreover, some researcher even combined it with Machine Learning to help improve recognition accuracy [7, 8].

We could not ignore the fact that morphology operation is one of the most popular techniques to be used in object recognition $[9,10]$. The reason for such popularity is that morphology operation while being not very complicated and costly; it could expose the whole structure of an object to ease the recognition of the desired object or to detect an undesired object to be removed from the image. Another essential technique is edge detection. Edge detection aims at finding the changes in brightness in an image to be used for capturing the critical event [11, 12]. The two of them are usually combined [13]. Although, some researchers have developed an object recognition algorithm using an edge detection and comparable using morphology operation $[14,15]$. This algorithm only works effectively when the border of the "object to be recognised" is strongly defined.

Another simplified approach to be used for object recognition is by using SIFT (scale-invariant feature transform), which proved to be robust against the change of scale and orientation [16]. It is known as the algorithm for object recognition. The algorithm works by utilising features in an image, known as critical 
points, to determine whether an object is similar or not. However, the algorithm may not be going to be very useful because of the lack of textures and features in the Indonesian license plate.

Following the research, [17] conducted a study to implement the algorithm for the Indonesian license plate. The algorithm still works effectively with some modification, especially in the pre-processing phase against various Indonesian license plate. Further research has been conducted by [18, 19]. Research [18] resulted that the implemented algorithm can recognise the characters on the police plate number in the average percentage $79,43 \%$. K-Nearest Neighbors algorithm reads all these characters. The test results show that this vehicle plate recognition system provides $83,33 \%$ success rate from 30 test data.

On the other hand, Damayanti et al. [19] using different approaches for the recognition of the license plate. The research utilises feature area and K-Nearest Neighbor (KNN) as a classification method. In summary, these results show that accuracy of $99.44 \%$.

\section{PROPOSED METHOD}

In this research, we proposed an improved Horizontal-Vertical Edge Projection algorithm. The preprocessing method proposed in this study has an unusual behaviour because it could reduce the intensity of specular reflection by a significant amount while highlighting the interest point, which is the vehicle license plate. Thus it could be very useful to settle the problems. The complete flow of the proposed algorithm could be seen in Figure 2.

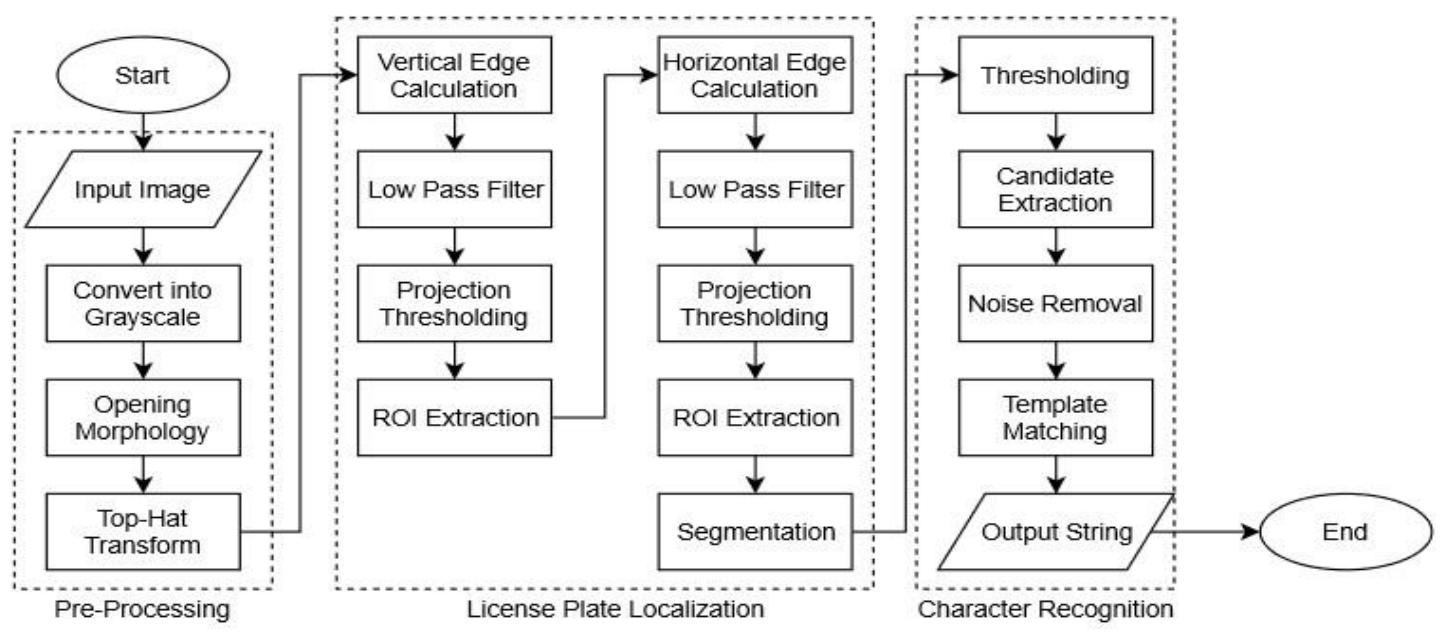

Figure 2. Flowchart of the proposed algorithm

\subsection{Pre-processing}

Pre-Processing is the phase to enhance the input image and remove unwanted data, noises, and distortion from the input image. The purpose of image preprocessing is to eliminate irrelevant information in the image, restore real useful information, enhance the detectability of the relevant information, and simplify the data to the maximum, thereby improving the reliability of image feature extraction and recognition. Common methods are: (1) grayscale; (2) geometric transformation; (3) image enhancement. The goal of this phase is to emphasise useful image information while reducing unwanted data. Furthermore, the data collected have many error or noise, so that it needs to perform the data preprocessing operation [20, 21].

\subsubsection{RGB to grayscale conversion}

The first step in the pre-processing phase is to convert the input image into the grayscale format. The reason behind this conversion is to simplify the computation process in the next stage. The most common technique to convert the RGB image into grayscale is to use the Luminosity method represented in (1) below. The result of this operation can be seen in Figure 3.

$$
\operatorname{Lum}_{(x, y)}=0.299 R_{(x, y)}+0.587 G_{(x, y)}+0.114 B_{(x, y)}
$$




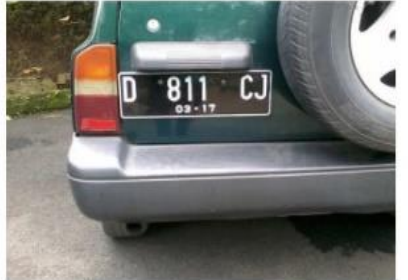

(a)

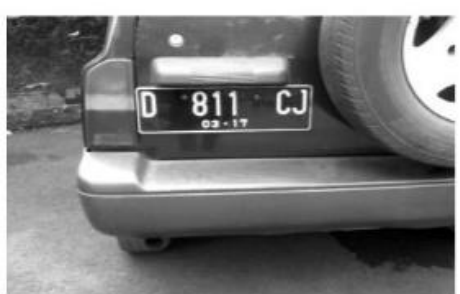

(b)

Figure 3. Comparison between, (a) original image and (b) grayscaled image

\subsubsection{Opening morphology operation}

The next step is to perform opening morphology operation to the image. Opening morphology could be achieved by implementing the erosion operation first, followed by the dilation operation using the same structuring element for both operations [22]. The information retrieved after this step is useful to detect problematic regions caused by having too much contrast/intensity or regions with specular reflection, which could confuse the license plate localisation algorithm. Opening operation is described by (2).

$$
A \circ B=(A \ominus B) \oplus B
$$

where $\ominus$ represents erosion operation while $\oplus$ represents dilation operation. The outcome of this operation is visualised by Figure .

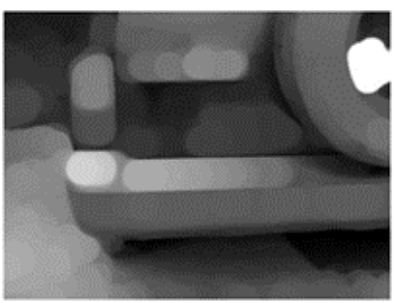

Figure 4. The result of opening morphology

\subsubsection{Top-hat transform}

Top-hat transform is one of the most used operations for image enhancement [22, 23]. In this case, the input image will be the grayscaled image, and the opening counterpart is the outcome of the previous step, which holds information about problematic regions as described before. The problematic regions will no longer dominate the image, and its intensity will be reduced to a significant amount. The region of interest (around the vehicle license plate) will be enhanced. This enhancement is what we needed to increase the accuracy of Horizontal-Vertical Edge Projection method in detecting the vehicle license plate. Figure 5 visualises the pre-processing phase proposed by Devapriya [5] and the proposed algorithm.

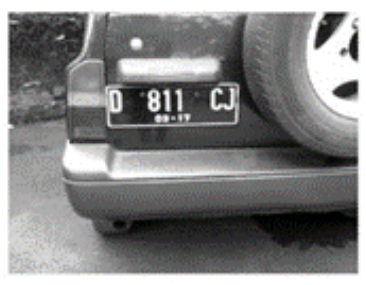

(a)

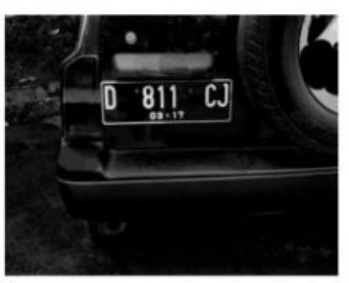

(b)

Figure 5. Comparison of pre-processing phase, (a) pre-processing as proposed by [3], (b) the proposed algorithm 


\subsection{License plate localisation}

After the enhanced image has been retrieved from the pre-processing phase, it is now the time to detect and localise the vehicle license plate. It means that the vehicle license plate should be identified precisely and isolated from other objects in the image.

\subsubsection{Vertical edge projection}

The enhanced image will be scanned vertically, and the sum of all magnitude differences will be calculated column-wise for every row of the image. All of the obtained value after the calculation will then be projected into a histogram representation for the analysis process. Figure 6 visualised the sample image and the histogram projection along the vertical edge.

The histogram should be smoothed using the low pass filter in order to normalise such drastic changes. The resulting values will be averaged to get a threshold level. The elimination process will yield to segmentation of the image to several rows based on the projection peaks. Figure 7 shows the comparison between each histogram projection for each operation.

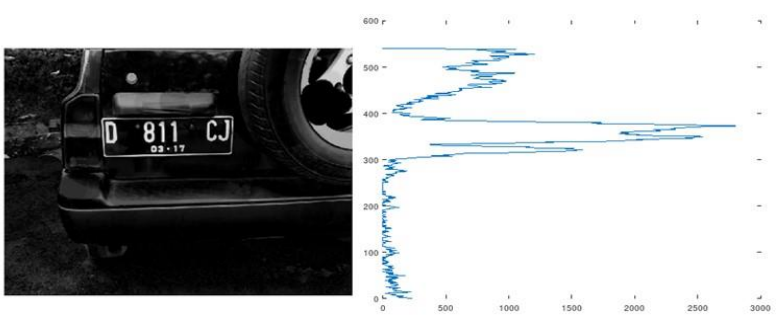

Figure 6. Vertical edge projection calculation

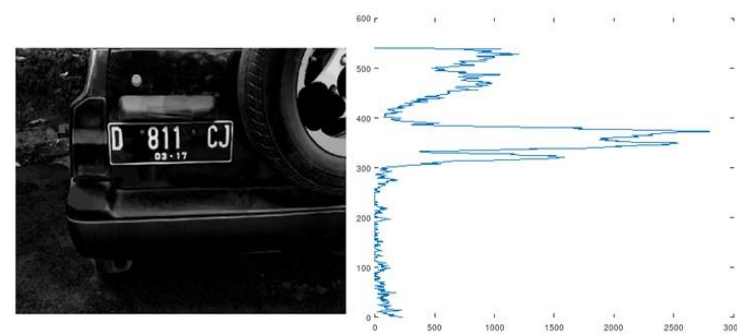

(a)

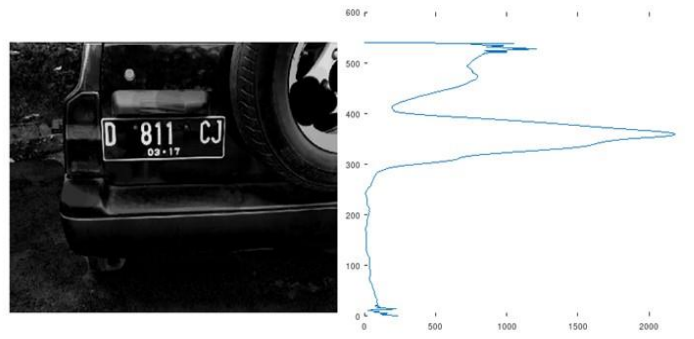

(b)

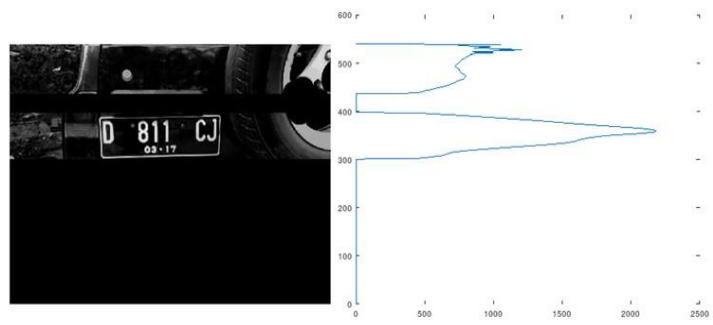

(c)

Figure 1. Vertical edge processing visualisation, (a) rough histogram projection, (b) low pass filter, (c) histogram projection thresholding 


\subsubsection{Region of interest extraction based on the vertical projection}

In this step, all the remaining peaks in the projection histogram will be analysed to find the region of interest. Since Indonesian license plate has a black coloured background with white coloured text and border, it must yield a significant amount of magnitude in the projection histogram. Therefore, the region of interest should be the peaks which contains a global maxima across the projection, and any other peaks should be eliminated from the image to localise the region of interest see Figure 8.
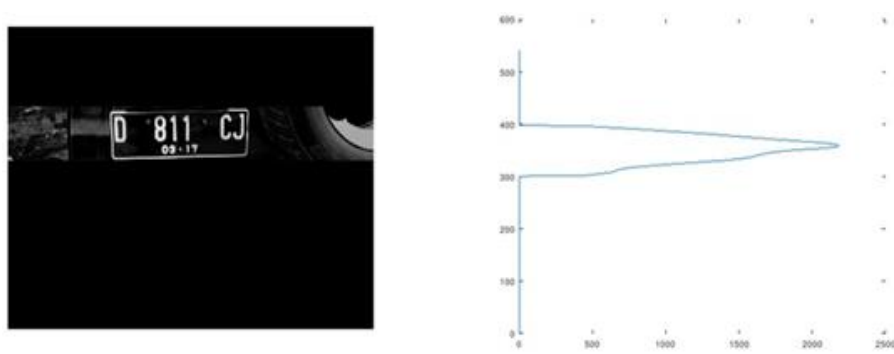

Figure 2. ROI based on the vertical edge

\subsubsection{Horizontal edge projection}

After the region of interest has been localised along the vertical edge, analysis along the horizontal edge is executed to precisely detect the vehicle license plate. The procedure to calculate the horizontal edge projection is identically the same as before, except the sum of all magnitude differences will be calculated between every neighbouring pixel in the vertical axis within such column. The outcome for each process is visualised by Figure 9.

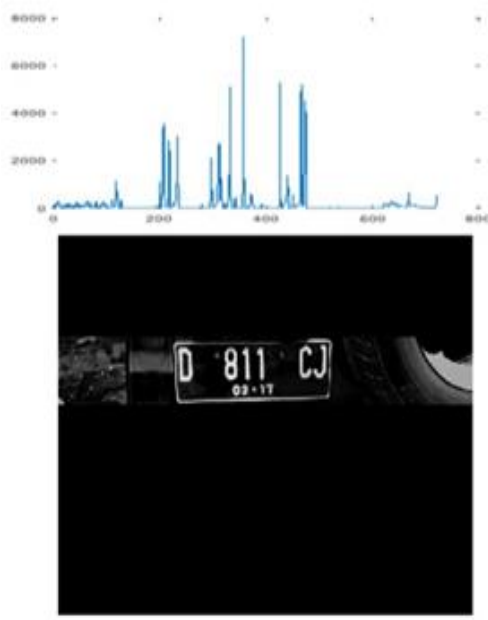

(a)

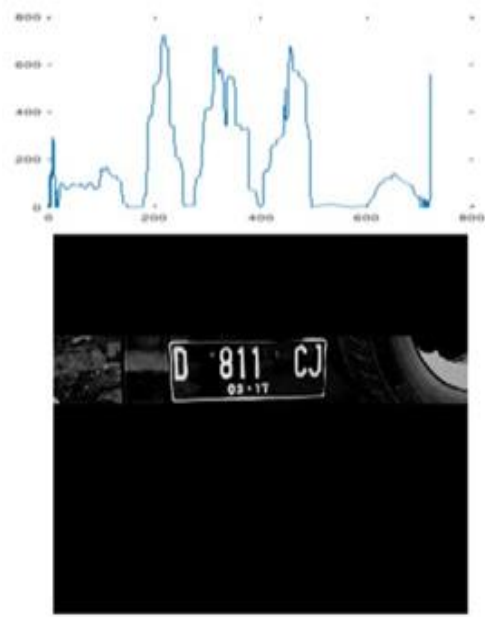

(b)

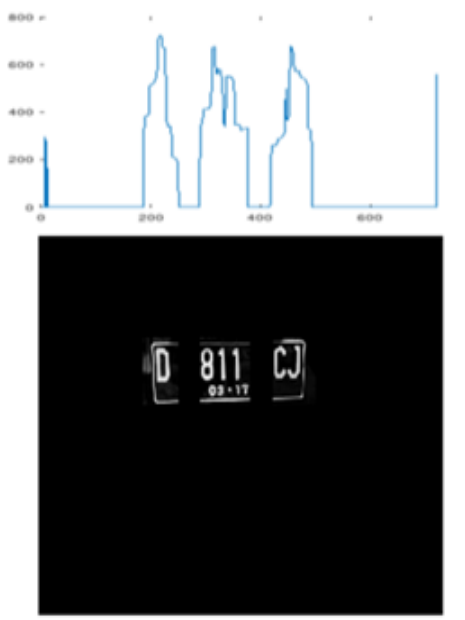

(c)

Figure 9. Horizontal edge processing visualisation, (a) rough histogram projection, (b) low pass filter, (c) histogram projection thresholding

\subsubsection{Region of interest extraction based on horizontal projection}

Different than before, the region of interest is analysed through the width of each peak in the projection histogram. We define a threshold value to filter out the peaks based on its width. For every peak having a width less than the threshold value will be considered as noise and must be eliminated, while those above the threshold value will be kept. In this research, we define the threshold value as 25 units see Figure 10. 


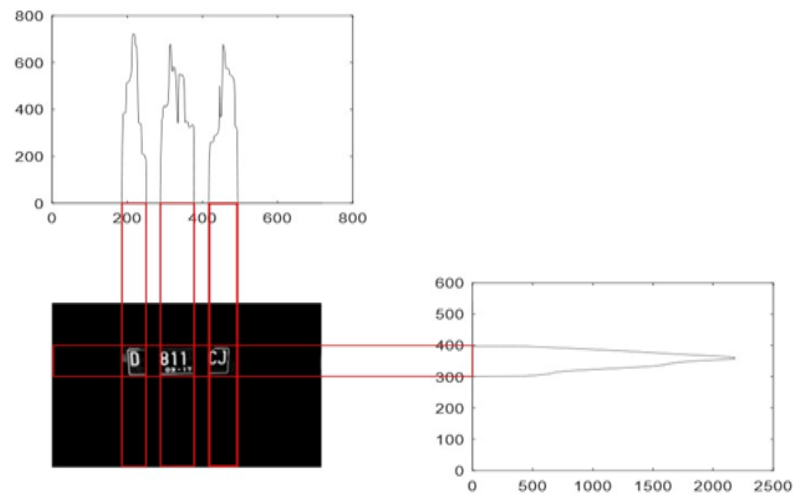

Figure 3. Final ROI

\subsubsection{License plate segmentation}

After the license plate has been precisely located and noises are successfully removed, the grayscaled image in the first phase will be cropped based on the region of interest defined from all of the previous steps see Figure 11. This cropped image will then be passed for the character recognition phase.

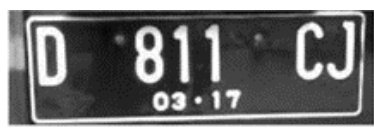

Figure 4. Cropped license plate

\subsection{Character recognition}

For character recognition phase, we modify the font template as recommended by Hidayatullah [4]. Instead, we use our own modified font template. This difference may affect the character recognition process.

\subsubsection{Thresholding (Otsu's method)}

The first step in character recognition phase is to convert the input image into a binary image by thresholding. The most commonly used method for thresholding is Otsu's method since it provides a dynamic threshold level which calculated from the image pixels $[24,25]$. This conversion means that for every pixel value below the threshold level will be converted into 0 (the darkest colour). In contrast, every pixel value above the threshold level will be converted into 1 (the brightest colour). The outcome of this conversion usually called binary image since it contains only 0 s and $1 \mathrm{~s}$ value in the pixel magnitude, as shown in Figure 12.

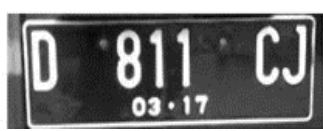

(a)

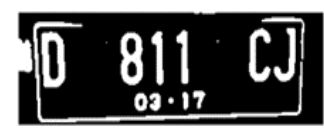

(b)

Figure 12. Comparison between, (a) grayscaled image and (b) binary image

\subsubsection{Character candidate extraction}

After the image has been converted into a binary image, we could quickly identify and extract all of the objects within the image through Connected Component Analysis. All of the detected objects are considered as character candidate as shown in Figure 13, however, some of them are better considered as noise. Therefore, further noise removal should be executed. Fortunately, Connected Component Analysis not only detect objects within an image but also assign some properties to the object such as centroids, and bounding box [26]. Enhancing the quality of binary image for plate image can be done based on preprocessed to remove the noise and compensate poor illumination [27]. Then, we can identify the position and the size of an object which could be useful for the noise removal process.

Noise removal process consists of several stages. The first stage is applying a median filter to remove salt and pepper noise. The second stage is eliminating objects whose size is below the defined 
threshold acquired from the equation proposed by [4]. The third stage is to eliminate objects which length exceed 1/8 length of the image. Furthermore, the last step is to check whether an object is parallel to the other object or not since the characters on a license plate are usually nearly parallel. After all of the license plate character candidates has been truly determined, they will be cropped out as an individual character. After that, they will be normalised by resizing them into $42 \times 24$ pixels to match the size of the font template. This cropping and normalisation process is required to perform template matching.

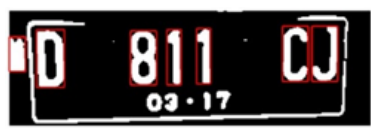

Figure 5. The visualisation of character candidate detection

\subsubsection{Template matching}

In this step, every individual character will be matched against sets of character template through such calculation to find similarity between them. In this research, we used the normalised cross-correlation method. Every calculation results obtained from the operation will be mapped inside an array for the voting process. The character is recognised as such string through the voting process by finding in which template the operation achieved maximum correlation value.

\section{EXPERIMENTAL RESULTS}

\subsection{License plate localisation}

To conduct a fair comparison between [3] and the proposed algorithm, we perform the research using the same sample images as used by them with their permission. The sample consists of 80 images, captured using various camera configurations such as the camera distance (between $1 \mathrm{~m}, 1.5 \mathrm{~m}$, or $2 \mathrm{~m}$ ), and height (between $55 \mathrm{~cm}, 1 \mathrm{~m}$, or $2 \mathrm{~m}$ ). Our proposed algorithm has successfully detected the vehicle license plate in 71 samples out of 80 samples, while [3] detect 63 samples out of 80 samples. Below is the table of comparison between [3] and the proposed algorithm.

Figure 14 presents the visualisation of the successful detection process for some samples, where the condition is not very optimal. Even though the proposed algorithm shows an improvement to the detection accuracy, it still finds struggles to detect the vehicle license plate in some samples. Some failed detection are shown in Figure 15. Figure $15(a, b)$ shows an extreme case where the detected license plate area exceeded the actual vehicle license plate due to the failure of the pre-processing phase to reduce the overly saturated car. This condition caused high projection value in the histogram and confused the analysis process. Figure 15 (c) shows the failure of the detection due to the usage of non-standardised license plate. The thinner font will not produce significant peaks in the projection histogram. Therefore, parts of the license plate did not exceed the threshold value in the horizontal projection analysis and get eliminated from the region of interest.

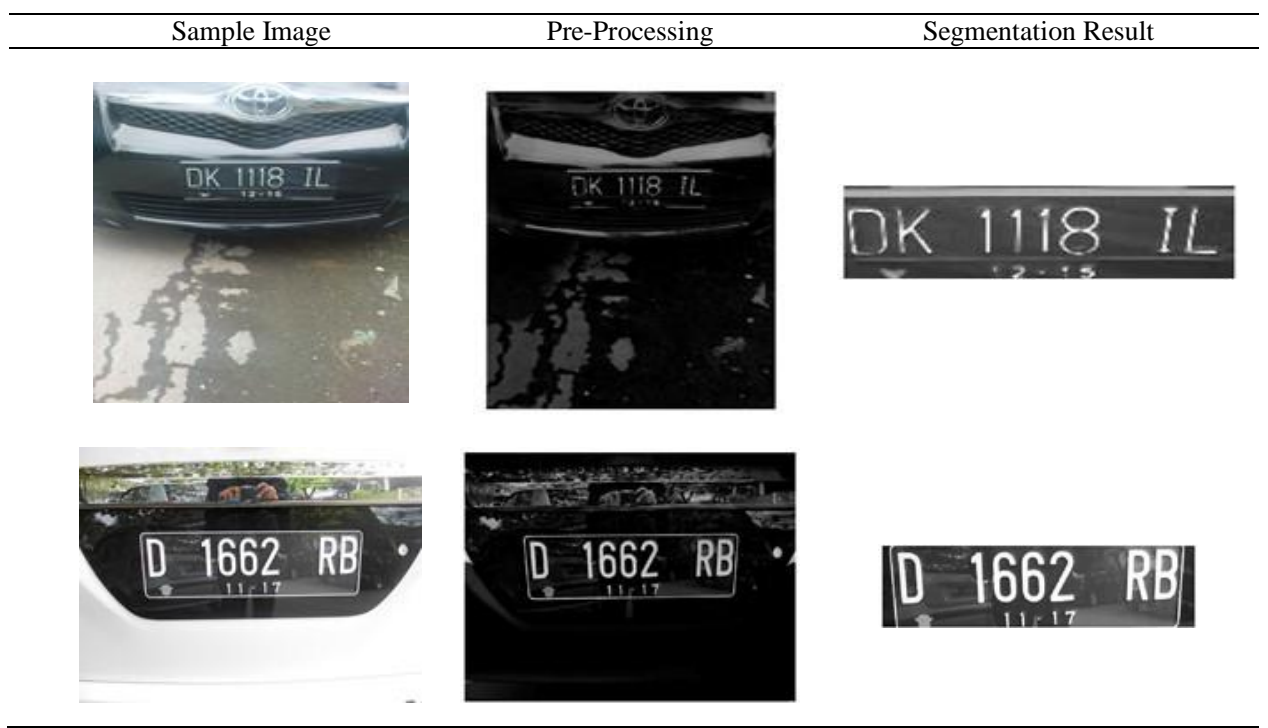

Indonesian J Elec Eng \& Comp Sci, Vol. 21, No. 2, February 2021 : 811 - 821 
Figure 14. (a) Camera a little higher than license plate, (b) case with specular reflection Sample Image Pre-Processing Segmentation Result
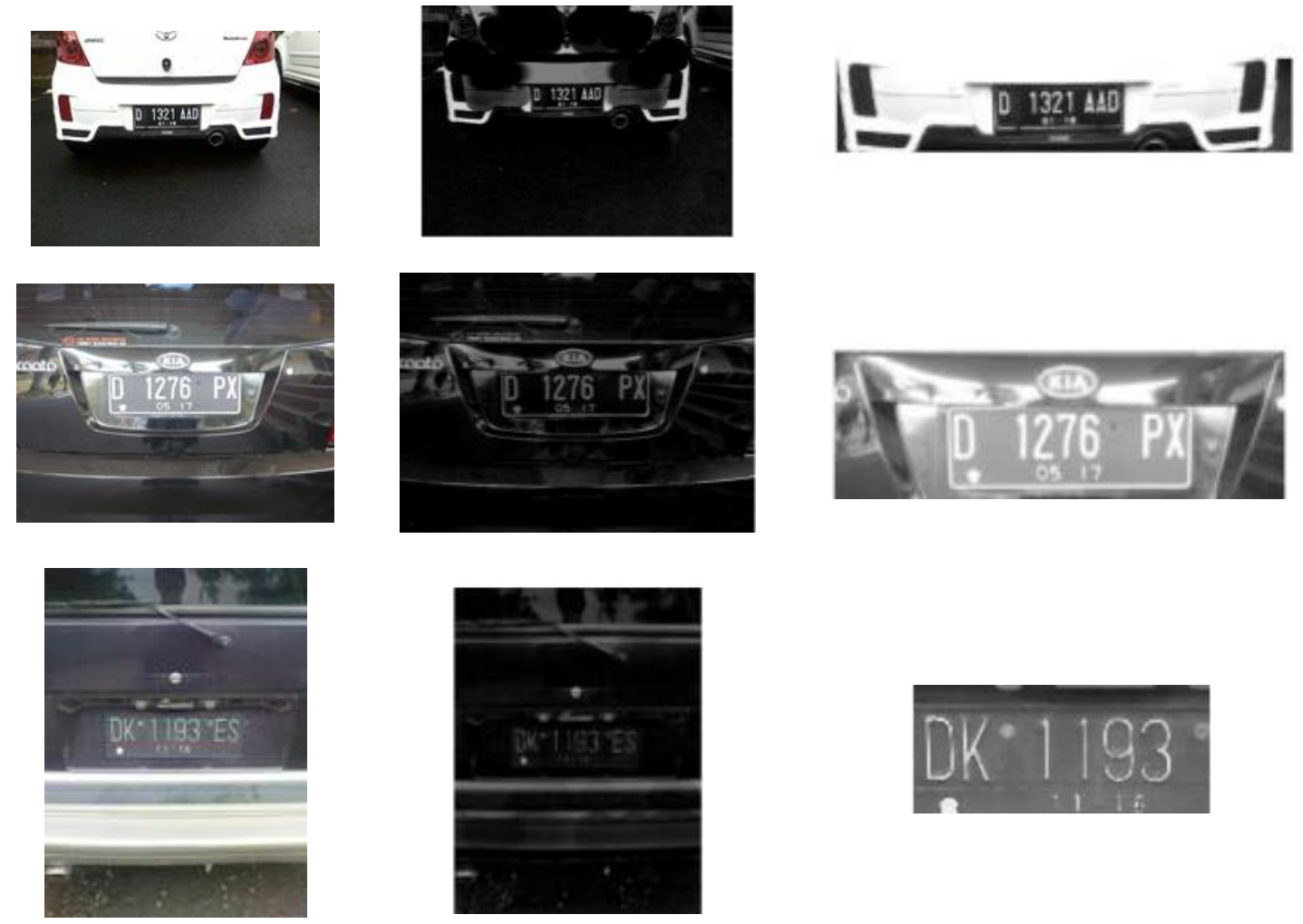

Figure 15. (a, b) license plate area exceeded, (c) license plate area cut

\subsection{Character recognition}

All of the 71 successfully detected license plates were then used for further processing which is the character recognition phase. Jaccard Similarity Index is used to calculate the similarity between the recognised text and the actual license plate. From the experiment, as shown in Table 1, we achieved an average of $77.97 \%$ similarity, while [3] obtained an average of $85.87 \%$ similarity. This gap might be caused by the presence of noise which falsely recognised as one of the license plate characters or the distortion among the license plate characters, which implies to false recognition. Table 2 shows the results of the character recognition stage.

Table 1. The comparison results in detection accuracy

\begin{tabular}{ccc}
\hline Algorithm & Detected sample (Out of 80 sample) & $\%$ \\
\hline Modified Horizontal-Vertical Projection & 63 & $78.75 \%$ \\
Proposed Agorithm & 71 & $88.75 \%$ \\
\hline
\end{tabular}

Table 2. The results of character recognition

\begin{tabular}{|c|c|c|c|c|}
\hline o. & Detection Result & Actual License Plate & Recognised & Similarity (\%) \\
\hline & 811 & D811CJ & D811CJ & 100 \\
\hline & 40 & D403LL & D403LL & 100 \\
\hline & $1732 \mathrm{WG}$ & D1732WG & D173G & 71.43 \\
\hline & 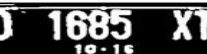 & D1685XT & $1 X$ & 28.57 \\
\hline & & D1311GW & - & 0 \\
\hline
\end{tabular}




\section{CONCLUSION}

The performance of Horizontal-Vertical Edge Projection could be improved by modifying the preprocessing phase to enhance the input image. Horizontal-Vertical Edge Projection is the simplest yet effective algorithm for license plate detection. Still, it suffers from the specular reflection problem because it analyses the region of interest-based on the magnitude value projection. Therefore, any object with high saturation intensity or specular reflection from the vehicle itself could confuse the analysis process, which often results in misdetection. Our research proposed an algorithm with the implementation to reduce such problems by doing an image enhancement in the pre-processing phase, which in result reducing the effect of specular reflection and object with high saturation intensity while preserving and enhancing the region of interest information. Our proposed algorithm has proven to be effective and improved the performance by a significant amount, from $63(78.75 \%)$ detected samples into $71(88.75 \%)$. The pre-processing phase sometimes still failed to reduce the specular reflection effect. For enhancement, sample image quality should also be taken care, blurry image and a dirty car could fail the localisation and recognition process. Another concern is that this algorithm is not very robust against orientation changes, and due to the limited sample we could not test the algorithm against the case when the camera is not perpendicular to the license plate.

\section{REFERENCES}

[1] S. Chakraborty and R. Parekh, "An Improved Template Matching Algorithm for Car License Plate Recognition," International Journal of Computer Applications, vol. 118, no. 25, pp. 16-22, 2015.

[2] Suzhi Zhang, Yuhong Wu, Jun Chang, "Survey of Image Recognition Algorithms", IEEE $4^{\text {th }}$ Information Technology, Networking, Electronic and Automation Control Conference, 2020.

[3] Sergio Montazzolli Silva, Claudio Rosito Jung, "Real-time license plate detection and recognition using deep convolutional neural networks", Journal of Visual Communication and Image Representation, 2020.

[4] P. Hidayatullah, F. Feirizal, H. Permana, Q. Mauluddiah, and A. Dwitama, "License plate detection and recognition for Indonesian cars," International Journal on Electrical Engineering Informatics, vol. 8, no. 2, pp. 331-346, 2016.

[5] W. Devapriya, C. N. K. Babu, and T. Srihari, "Indian License Plate Detection and Recognition Using Morphological Operation and Template Matching," International Journal of Computer, Electrical, Automation, Control and Information Engineering, vol. 9, no. 4, pp. 1049-1055, 2015.

[6] Naikur B. Gohil, “Car License Plate Detection”, Sacramento, California State University, 2010.

[7] Hui Li, Peng Wang, and Chunhua Shen, "Toward End-to-End Car License Plate Detection and Recognition With Deep Neural Networks”, IEEE Transactions On Intelligent Transportation Systems, pp 99, 2017.

[8] Naaman Omar, Abdulkadir Sengur, Salim Ganim Saeed Al-Ali, "Cascaded deep learning-based efficient approach for license plate detection and recognition", Expert Systems With Applications, 2020.

[9] Zhongli Wang, Xiping Ma and Wenlin Huang, "Vehicle License Plate Recognition Based on Wavelet Transform and Vertical Edge Matching”, International Journal of Pattern Recognition and Artificial Intelligence, vol. 34, no. 6, 2020.

[10] K. Adi, A. P. Widodo, C. E. Widodo, A. Pamungkas, and A. B. Putranto, "Automatic vehicle counting using background subtraction method on gray scale images and morphology operation," Journal of Physics: Conference Series, vol. 1025, no. 1, 2018.

[11] Elena Medvedeva, Igor Trubin, and Pavel Kasper, "Vehicle License Plate Recognition Based on Edge Detection", Proceeding of the $26^{\text {th }}$ Conference of Fruct Association, 2020.

[12] S. Mahalakshmi and P. M. Karani, "Study of Edge Detection Techniques in Automatic License Plate Recognition (ALPR)," International Research Journal of Engineering and Technology, vol. 4, no. 5, pp. 1658-1661, 2017.

[13] S. Borker, "Implementation of Vehicle License Plate Recognition Using Canny Edge Detection," International Journal of Computer Science and Engineering Technology (IJCSET), vol. 6, no. 06, pp. 376-379, 2015.

[14] Chen, Y, Luo, S., "Research on License Plate Location in Different Scenes", International Conference on Artificial Intelligence and Advanced Manufacturing, 2019.

[15] Nurhaida, A. Noviyanto, R. Manurung, and A. M. Arymurthy, “Automatic Indonesian's Batik Pattern Recognition Using SIFT Approach,” Procedia Computer Science, vol. 59, pp. 567-576, 2015.

[16] F. A. Hermawati and R. Koesdijarto, "a Real-Time License Plate Detection System for Parking Access," TELKOMNIKA (Telecommunication, Computing, Electronics and Control), vol. 8, no. 2, pp. 97-106, 2010.

[17] Rahmat, B., Joelianto, E., Ketut Eddy Purnama, Purnomo, M.H., "Vehicle license plate image segmentation system using cellular neural network optimized by adaptive fuzzy and Neuro-Fuzzy algorithms", International Journal of Multimedia and Ubiquitous Engineering, vol. 11, no. 12, pp. 383-400, 2016.

[18] Fitri Damayanti, Sri Herawati, Imamah, Fifin Ayu M, Aeri Rachmad, "Indonesian license plate recognition based on area feature extraction", TELKOMNIKA (Telecommunication, Computing, Electronics and Control), vol.17, no. 2, pp. 620-627, 2019.

[19] V. Ayumi, L. M. R. Rere, M. I. Fanany, and A. M. Arymurthy, "Random Adjustment - Based Chaotic Metaheuristic Algorithms for Image Contrast Enhancement," Journal of a Science and Information, vol. 10, no. 2, pp. 67-76, 2017.

[20] S. Salazar-Colores, J. M. Ramos-Arreguín, C. J. Ortiz Echeverri, E. Cabal-Yepez, J. C. Pedraza-Ortega, and J. Rodriguez-Resendiz, "Image dehazing using morphological opening, dilation and Gaussian filtering," Signal, Image Video Process., vol. 12, no. 7, pp. 1329-1335, 2018. 
[21] Mujiono Sadikin, Fahri Alfiandi, "Comparative Study of Classification Method on Customer Candidate Data to Predict its Potential Risk", International Journal of Electrical and Computer Engineering (IJECE), vol. 8, no. 6, pp. 4763-4771, 2018.

[22] J. C. M. Roman, H. L. Ayala, and J. L. V. Noguera, "Top-Hat Transform for Enhancement of Aerial Thermal Images," 2017 30th SIBGRAPI Conference on Graphics, Patterns and Images (SIBGRAPI), Niteroi, pp. 277-284, 2017.

[23] J. C. Mello Román, J. Luis Vázquez Noguera, H. Legal-Ayala, L. Moré, and D. P. Pinto-Roa, "Image enhancement with preservation of brightness and details using multiscale top-hat transform," 2019 XXII Symposium on Image, Signal Processing and Artificial Vision (STSIVA), Bucaramanga, Colombia, pp. 1-5, 2019.

[24] Feng, Y., Zhao, Li, X., Zhang, X., Li, H., "A multi-scale 3D Otsu thresholding algorithm for medical image segmentation", Digital Signal Processing: A Review Journal, vol. 60, pp. 186-199, 2017.

[25] Mohamed Abd El Aziz, Ahmed A. Ewees, and Aboul Ella Hassanien, "Whale Optimization Algorithm and MothFlame Optimization for Multilevel Thresholding Image Segmentation”, Expert Systems With Applications, vol. 83, pp. 242-256, 2017.

[26] L. He, X. Ren, Q. Gao, X. Zhao, B. Yao, and Y. Chao, "The connected-component labelling problem: A review of state-of-the-art algorithms," Pattern Recognition, vol. 70, pp. 25-43, 2017.

[27] Sajed Rakhshani, Esmat Rashedi, Hossein Nezamabadi, "Representation learning in a deep network for license plate recognition”, Multimedia Tools and Applications, vol. 79, no. 19-20, pp. 13267-13289, 2020.

\section{BIOGRAPHIES OF AUTHORS}
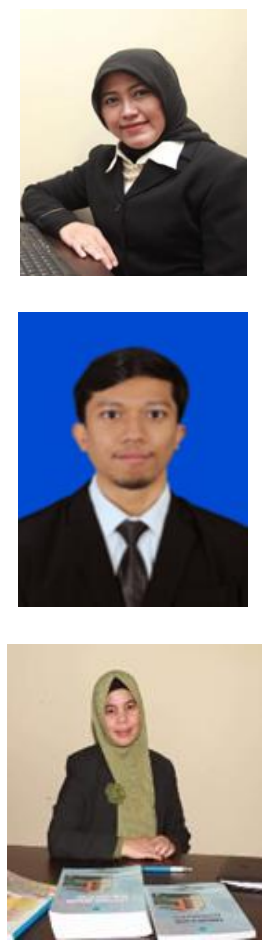

Ida Nurhaida was born in Kuantan (Malaysia) in 1971. She is a researcher and member of Faculty of Computer Science Universitas Mercu Buana, Indonesia. Her areas of interest and research are in the image processing, pattern recognition and image retrieval system which she formalised in her PhD (2010) on this subject in the Faculty of Computer Science, University of Indonesia. She has presented papers at conferences both home and abroad, published articles and papers in various journals, and contributed a chapter to the book.

Imam Nududdin was born in Jakarta (Indonesia) in 1998. He received his bachelor degree in Computer Science from Universitas Mercu Buana, Indonesia in 2020. His research interest are image processing, pattern recognition, and computer vision.

Desi Ramayanti was born in Padang (Indonesia) in 1981. She is a researcher and member of Faculty of Computer Science Universitas Mercu Buana, Indonesia. Her areas of interest and research are in machine learning, networking and software development which she formalised in her Master (2008) on this subject in Computer Engineering Institute of Technology Bandung. 